\title{
Impact of Galcanezumab on Total Pain Burden: A Post Hoc Analysis of a Phase 3, Randomized, Double-Blind, Placebo-Controlled Study in Patients with Episodic Cluster Headache
}

\author{
J Scott Andrews' \\ David Kudrow ${ }^{2}$ \\ Mallikarjuna Rettiganti \\ Tina Oakes' \\ Jennifer N Bardos' \\ Richard Wenzel' \\ Dulanji K Kuruppu' \\ Charly Gaul ${ }^{3}$ \\ James M Martinez' \\ 'Eli Lilly and Company, Indianapolis, IN, \\ USA; ${ }^{2}$ California Medical Clinic for \\ Headache, Santa Monica, CA, USA; \\ ${ }^{3}$ Headache Center, Frankfurt, Germany
}

\begin{abstract}
Purpose: In a phase 3 study, galcanezumab significantly reduced the frequency of episodic cluster headache attacks across weeks 1-3 (primary endpoint) compared with placebo. However, multiple pain dimensions may contribute to the total burden of episodic cluster headache pain. This post hoc analysis assessed the impact of galcanezumab on the total pain burden of episodic cluster headache using a composite measure.

Patients and Methods: Patients with episodic cluster headache were randomized 1:1 to galcanezumab $300 \mathrm{mg}$ or placebo once monthly for 8 weeks. Mean weekly total pain burden was calculated (daily cluster headache attack frequency $\times$ average duration $\times$ average pain severity summed over 7 days) using data collected in an electronic patient-reported outcomes diary. Change from baseline in weekly total pain burden across weeks 1-3 was compared between galcanezumab and placebo. To explore construct validity, mean weekly total pain burden scores were stratified by Patient Global Impression of Improvement (PGI-I) responses at the week 4 clinic visit.
\end{abstract}

Results: The reduction from baseline in mean weekly total pain burden was significantly greater with galcanezumab $(\mathrm{N}=49)$ than with placebo $(\mathrm{N}=57)$ : the least squares mean difference was -11.18 severity-weighted hours $(\mathrm{p}=0.035)$. Median weekly total pain burden decreased as PGI-I ratings improved, from 33.6 to 5.0 severity-weighted hours for patients who felt "very much worse" and "very much better," respectively.

Conclusion: Galcanezumab significantly reduced mean weekly total pain burden compared with placebo in patients with episodic cluster headache. The composite pain measure demonstrated construct validity. Total pain burden may provide a holistic measure of the pain of episodic cluster headache.

Clinical Trials: ClinicalTrials.gov, NCT02397473.

Keywords: composite pain, frequency, severity, duration, CGRP

\section{Introduction}

Episodic cluster headache $(\mathrm{CH})$ is a debilitating disease characterized by daily attacks of intense unilateral headache with autonomic symptoms such as lacrimation, nasal congestion, forehead and facial sweating, restlessness, and agitation. ${ }^{1}$ The headache attacks occur over periods lasting from 7 days to 1 year and are separated by pain-free periods of at least 3 months. ${ }^{1}$ Patients with episodic $\mathrm{CH}$ can experience multiple, sometimes excruciatingly painful, headache attacks each day, lasting from 15 minutes to 3 hours. ${ }^{1}$ The pain associated with $\mathrm{CH}$ has been
Correspondence: Mallikarjuna Rettiganti Eli Lilly and Company, Lilly Corporate Center, 893 Delaware St., Indianapolis, IN, 46285, USA

$\mathrm{Tel}+$ I 31765|9378

Email rettiganti_mallikarjuna@lilly.com 
described as one of the most severe types of pain experienced by humans; ${ }^{2}$ consequently, the disease has a significant negative impact on patients' health-related quality of life. ${ }^{3,4}$ The periods of $\mathrm{CH}$ attack can increase the risk of self-injurious behavior ${ }^{5}$ and suicidal ideation. ${ }^{6}$ $\mathrm{CH}$ also poses a significant economic burden due to absenteeism and presenteeism. ${ }^{7-10}$

The realistic goal of treatment for episodic $\mathrm{CH}$ is to shorten the cluster period and to reduce the frequency and/or severity of $\mathrm{CH}$ attacks. ${ }^{11,12}$ Guidelines recognize the need for both acute (abortive) treatment (treatment taken at the start of a headache attack to shorten the attack duration and reduce its severity) and preventive treatment (prophylaxis; treatment to reduce the frequency of headache attacks, starting as early as possible after the start of a new cluster period). ${ }^{11-16}$ For acute treatment, subcutaneous or intranasal sumatriptan and high-flow oxygen are recommended. ${ }^{11-16}$ For preventive treatment, verapamil and lithium are among the treatment options used. ${ }^{16}$ Some preventive treatments (such as suboccipital corticosteroid injections or oral steroids), known as transitional treatments, are reserved for shortterm use or for "bridge" therapy to provide rapid relief, while another preventive treatment (such as verapamil) takes effect. ${ }^{16}$

Galcanezumab is a humanized monoclonal antibody that selectively binds to and inhibits the biological activity of calcitonin gene-related peptide (CGRP), a peptide involved in the pathophysiology of $\mathrm{CH}$ and migraine. ${ }^{17-}$ ${ }^{21}$ Galcanezumab has been approved by the US Food and Drug Administration (FDA) for the treatment of episodic $\mathrm{CH}$ in adults $^{22}$ and for the preventive treatment of migraine. ${ }^{23-28}$

In a phase 3 study of the efficacy and safety of galcanezumab for the preventive treatment of episodic $\mathrm{CH}$, the frequency of $\mathrm{CH}$ attacks across weeks 1-3 (primary endpoint) was significantly reduced compared with placebo. ${ }^{29}$ Since several dimensions of pain may contribute to the overall pain burden experienced during $\mathrm{CH}$ attacks, including frequency, duration, and severity, ${ }^{30-32}$ a composite measure that incorporates multiple pain dimensions may prove useful to quantify the pain burden experienced. ${ }^{30}$ As such, a post hoc analysis of results from the phase 3 study $^{29}$ was performed to describe the total pain burden associated with episodic $\mathrm{CH}$ and to assess the impact of galcanezumab on this burden. Construct validity of the composite outcome and total pain burden was also evaluated.

\section{Patients and Methods}

\section{Study Design}

This post hoc analysis was based on data from a phase 3 , randomized, double-blind, placebo-controlled study of galcanezumab in patients with episodic $\mathrm{CH}$ treated in the outpatient setting by licensed physicians with a specialty in neurology and headache (NCT02397473). ${ }^{29}$ The study, conducted at 35 sites in Europe and North America, consisted of a screening/washout phase lasting for a minimum of 0 days (for patients in an active cluster period and not receiving medications that required washout) to a maximum of 12 months (for patients who entered screening while in remission and were awaiting the next cluster period before being eligible to enter into the baseline phase). A prospective baseline phase started on the day patients first recorded a $\mathrm{CH}$ attack in their electronic patient-reported outcome (ePRO) diary and lasted for 10 to 15 days, during which time eligibility and baseline weekly $\mathrm{CH}$ characteristics were determined over 7 consecutive days. This was followed by an 8-week, randomized, double-blind, placebo-controlled treatment period and 16 weeks of post-treatment washout (Figure 1). Eligible patients were randomized 1:1 using a computer-generated random sequence and an interactive Web-response system to receive galcanezumab $300 \mathrm{mg}$ or placebo subcutaneously once monthly for 8 weeks. Investigators and participants were blinded to the intervention received. Further details of the study and the primary results are presented elsewhere. $^{29}$

The study was conducted in accordance with ethical guidelines of the Declaration of Helsinki and the Council for International Organizations of Medical Sciences and the study protocol was approved by an appropriate ethical review board for each investigative site (Supplementary Table 1). All patients provided written informed consent.

\section{Patients}

The study included male or female outpatients aged 18-65 years with a diagnosis of episodic $\mathrm{CH}$ as defined by International Classification of Headache Disorders (ICHD)-3 beta criteria. ${ }^{33}$ During 7 consecutive days of the prospective baseline period, patients had to experience a total of four or more $\mathrm{CH}$ attacks, an attack frequency of a minimum of one attack every other day, and no more than eight attacks per day to be eligible for randomization. Patients were also expected to have a prior history of a cluster period lasting for $\geq 6$ weeks and to be able to 


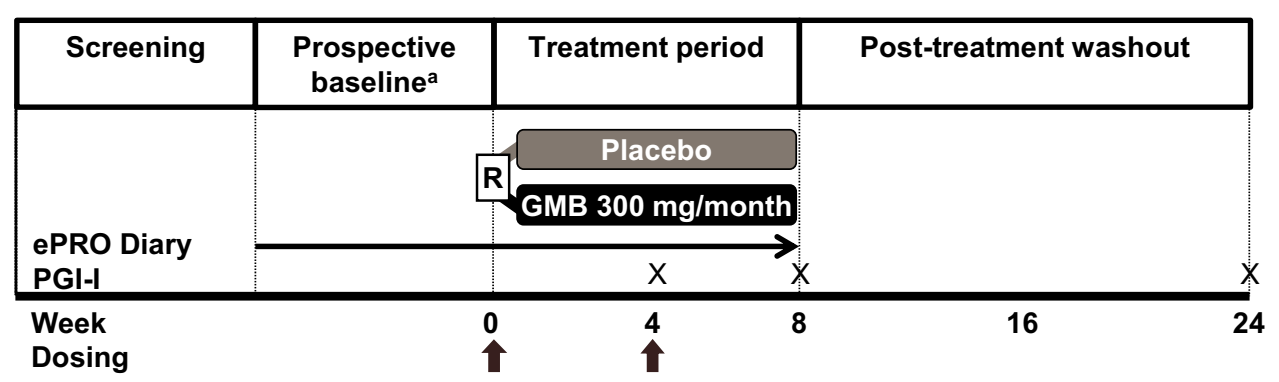

Figure I Design of the phase 3, randomized, double-blind, placebo-controlled study of galcanezumab in patients with episodic CH (NCT02397473). ${ }^{28}$ a Prospective baseline period started on the day the patient first recorded a $\mathrm{CH}$ attack in their ePRO diary. Eligibility and baseline weekly $\mathrm{CH}$ characteristics were assessed over 7 consecutive days during the prospective baseline period.

Abbreviations: $\mathrm{CH}$, cluster headache; ePRO, electronic patient-reported outcome; GMB, galcanezumab; PGI-I, Patient Global Impression of Improvement; R, randomization; $\mathrm{X}$, administration of PGI-I at clinic visit.

distinguish $\mathrm{CH}$ attacks from other types of headache. Patients were allowed to use the following concomitant acute treatments throughout the study: high-flow oxygen, oral triptans (after a protocol amendment), sumatriptan subcutaneous, sumatriptan or zolmitriptan nasal spray, acetaminophen, and nonsteroidal anti-inflammatory drugs. Treatment guidelines do not usually recommend oral triptans as acute treatment for $\mathrm{CH}$ attacks due to the slower onset of effect compared to subcutaneous and intranasal formulations. ${ }^{11-16}$ Oral triptans were therefore originally excluded from the list of permitted acute treatments. However, they were later introduced as an eligible acute treatment by protocol amendment to allow patients who preferred oral triptans to treat $\mathrm{CH}$ attacks as they would in routine practice. Concomitant preventive treatments for $\mathrm{CH}$ were not allowed.

Key exclusion criteria were recent participation in a clinical trial of an investigational drug or device; current or any previous use of any CGRP antibody, antibody to the CGRP receptor, or antibody to nerve growth factor; concurrent use of other therapeutic monoclonal antibodies; and having another distinct trigeminal autonomic cephalalgia or a history of migraine variants that could have been due to cerebral ischemia.

\section{Outcome Measures for the Current Analysis}

During the prospective baseline and double-blind treatment periods, patients completed an ePRO diary on a daily basis (based on a 24-hour period) in which they recorded the daily number of $\mathrm{CH}$ attacks, the average daily duration of the attacks (options in the ePRO diary for patients to choose from were 15 or 30 minutes, or 1, 2, 3 hours, or $>3$ hours), and the average severity of attacks, measured on a 5 -point scale, where $0=$ no pain, $1=$ mild pain, $2=$ moderate pain, $3=$ severe pain, and $4=$ very severe pain. Patients were requested to record all cluster attacks regardless of attack duration. At the scheduled clinic visit at week 4, patients were also asked to complete the Patient Global Impression of Improvement (PGI-I), a global index that captures patient perception of improvement in their disease using a 7-point scale, where $1=$ very much better, $2=$ much better, $3=\mathrm{a}$ little better, $4=$ no change, $5=\mathrm{a}$ little worse, $6=$ much worse, and $7=$ very much worse. Patients were asked to select the category that best described their $\mathrm{CH}$ since they started taking study medication.

\section{Statistical Analysis Individual Pain Components}

The effect of galcanezumab on each individual $\mathrm{CH}$ attack component (frequency, duration, severity) was analyzed. The primary study outcome measure was the change from baseline in weekly $\mathrm{CH}$ attack frequency. Weekly mean duration per $\mathrm{CH}$ attack was computed as follows. First, for each patient, the daily total duration of $\mathrm{CH}$ attacks was estimated as the product of the daily average duration of each attack and the daily frequency of attacks. This was repeated for each day of the week and summed for all days of the week to obtain the weekly total duration of $\mathrm{CH}$ attacks. The weekly mean duration per $\mathrm{CH}$ attack was then computed as the ratio of weekly total duration of $\mathrm{CH}$ attacks divided by the weekly $\mathrm{CH}$ attack frequency. The mean severity of weekly $\mathrm{CH}$ attacks was calculated as the mean of all daily average severity scores in a week for weeks with at least one $\mathrm{CH}$ attack. Both weekly mean duration per attack and the mean severity of remaining weekly attacks were defined only on weeks with at least one $\mathrm{CH}$ attack; thus, patients with no $\mathrm{CH}$ attacks in each of the first 3 weeks were excluded from the analyses. 


\section{Calculation of Composite Score}

A composite pain measure was developed that combined the three different facets of pain experienced by patients with $\mathrm{CH}$ (attack frequency, duration, and severity) into one clinical outcome assessment called the total pain burden. This composite outcome was compared between the two treatment groups to assess whether galcanezumab had a greater impact on $\mathrm{CH}$ total pain burden than did placebo.

The daily pain burden, expressed in severity-weighted hours, was first calculated for each patient by multiplying the daily frequency of attacks by the average duration of attacks (in hours) in a day and by the average pain severity of attacks in a day. This value was then summed over a weekly period to give the weekly total pain burden:

Weekly total pain burden (severity-weighted hours) $=$ sum over week (daily frequency of attacks $\times$ daily average duration of attacks [hours] $\times$ average daily attack pain severity)

Patients who experienced no $\mathrm{CH}$ attacks in a week were assumed to have zero total pain burden. Attack duration and pain severity represented patients' average over 24 hours regardless of any acute medication used (ie, they were not collected on a per attack basis and later averaged). The scores representing pain severity in the composite score calculation were applied from the prespecified 5-point pain scale, with severity scores of 0, 1, 2, 3, and 4 reflecting no, mild, moderate, severe, and very severe pain, respectively. To analyze the impact of using different weights for the severity categories 1 to 4 on the treatment effect, sensitivity analyses were conducted in which total pain burden was calculated using the square root of attack severity scores (ie, weights of 1.000, 1.414, 1.732, and 2.000 for the four categories of attack severity) or the square of attack severity scores (ie, weights of 1, 4, 9 , and 16 for the four categories of attack severity). As for the main analyses, patients who experienced no $\mathrm{CH}$ attacks in a week were assumed to have zero total pain burden; as this is not likely to be considered subjective, it was excluded from the sensitivity analyses.

Mean changes from baseline in each individual pain component and in total pain burden, and the percent change from baseline in total pain burden across weeks 1-3 were compared between galcanezumab and placebo using mixed-model repeated measures (MMRM) analyses adjusted for baseline value, sex, pooled investigative site, week, and treatment-by-week interaction. Analyses of the mean severity of $\mathrm{CH}$ attacks included baseline $\mathrm{CH}$ attack category (up to four attacks vs more than four attacks per day) as an additional variable in the model.

\section{Construct Validity}

The composite outcome of total pain burden was assessed for construct validity by relating it to the PGI-I responses. Average weekly total pain burden scores across weeks 1-3 were stratified by PGI-I responses at week 4 using pooled data from galcanezumab- and placebo-treated patients. In addition, logistic regression analysis was used to predict the impact of average weekly total pain burden scores across weeks $1-3$ on the dichotomous PGI-I score at week 4 of "feeling better" (PGI-I score 1, 2, or 3, representing "very much better," "much better," or "a little better," respectively) or "not feeling better" (PGI-I score $4,5,6$, or 7, representing "no change," "a little worse," "much worse," or "very much worse," respectively). Results were expressed as an odds ratio (OR) with 95\% confidence interval (CI) for "feeling better" for every 10unit decrease in average total pain burden across weeks $1-3$.

\section{Contribution of Individual Pain Components to Total Pain Burden}

The magnitude and impact of each pain component on the total pain burden score was assessed in two ways. First, using baseline data, the strength of association between each individual pain component and total pain burden was evaluated using Spearman's rank correlation. The proportion of unique variability explained by each individual $\mathrm{CH}$ attack component (frequency, duration, and severity) in total pain burden variability was assessed using squared semi-partial type II correlations. These were estimated using a linear regression model on the log-transformed variables, with total pain burden as the outcome and the individual components weekly $\mathrm{CH}$ attack frequency, weekly average attack duration, and weekly average attack severity as predictors. These partial correlations gave an estimate of the proportion of variability in total pain burden explained by each component after including/adjusting for the other two components. In this analysis, if the weekly frequency of attacks was 0 , duration per attack and average severity were also assumed to be 0 .

Second, to assess whether the treatment effect observed in total pain burden was primarily due to the treatment effect on reduction in weekly $\mathrm{CH}$ attack frequency, we analyzed change from baseline in weekly total pain burden in the first 3 weeks of the double-blind treatment phase 
while additionally adjusting for the effect of change from baseline in weekly $\mathrm{CH}$ attack frequency. This was achieved using an MMRM model that included the fixed effects of baseline total pain burden, treatment group, sex, pooled investigative site, week, and treatment-by-week interaction, with change from baseline in weekly $\mathrm{CH}$ attack frequency as a time-varying covariate.

\section{General Considerations}

All analyses were conducted on the intent-to-treat population, which included all patients who were randomized and received at least one dose of study drug. Summary statistics are presented using mean and standard deviation or median and quartiles for continuous variables. Counts and percentages were used to summarize categorical variables. For the change from baseline analyses, only patients who had both a baseline measurement and at least one valid post-baseline measurement were included. All MMRM models assumed an unstructured variance covariance matrix to estimate the correlation between repeated measures on the same subject. For all MMRM models, mean changes from baseline were estimated using least squares (LS) means and standard errors (SEs), and treatment effects were estimated using LS mean differences between treatment groups and 95\% CIs. Unless otherwise specified, all analyses were post hoc in nature and no adjustments for multiple testing were made; consequently, all results should be considered exploratory. Analyses were conducted using SAS ${ }^{\circledR}$ Enterprise Guide version 9.4, assuming a two-sided significance level of $5 \%$.

\section{Results}

\section{Baseline Characteristics}

Enrollment in the primary study was stopped before the planned sample size had been reached due to lower-thananticipated numbers of patients entering an active $\mathrm{CH}$ period during the screening period. The first patient visit took place in May 2015 and the last patient visit in June 2018. Data were available for analysis from 106 patients, of whom 49 received galcanezumab and 57 received placebo. A figure of patient flow through the study is presented in the primary publication. ${ }^{29}$ Baseline demographics and disease characteristics - including mean weekly use of acute medications (pooled), high-flow oxygen, and subcutaneous sumatriptan, and mean baseline total weekly pain burden scores-were not statistically significantly different between the two treatment groups (Table 1).
Table I Baseline Demographics and Disease Characteristics of Patients Included in the Post Hoc Analyses

\begin{tabular}{|c|c|c|}
\hline Characteristic & $\begin{array}{l}\text { Galcanezumab } \\
(\mathrm{N}=49)\end{array}$ & $\begin{array}{l}\text { Placebo } \\
(N=57)\end{array}$ \\
\hline Age, years & $47.5 \pm 10.7$ & $45.4 \pm 11.3$ \\
\hline Male & $41(83.7)$ & $47(82.5)$ \\
\hline $\begin{array}{l}\text { Use of acute medication }^{\text {a }} \\
\text { Use of high-flow oxygen } \\
\text { Use of s.c. sumatriptan }\end{array}$ & $\begin{array}{l}17.1 \pm 15.1 \\
9.5 \pm 9.6 \\
9.1 \pm 10.8\end{array}$ & $\begin{array}{l}16.9 \pm 14.9 \\
9.2 \pm 9.5 \\
9.1 \pm 7.2\end{array}$ \\
\hline \multicolumn{3}{|c|}{ Baseline $\mathrm{CH}$ characteristics } \\
\hline $\begin{array}{l}\text { Weekly attack frequency } \\
\text { Weekly attack duration, } \\
\text { hours } \\
\text { Weekly attack severity }\end{array}$ & $\begin{array}{l}17.8 \pm 10.1 \\
15.9 \pm 16.0 \\
2.5 \pm 0.7\end{array}$ & $\begin{array}{l}17.3 \pm 10.1 \\
15.1 \pm 13.2 \\
2.6 \pm 0.7\end{array}$ \\
\hline \multicolumn{3}{|c|}{ Baseline weekly total pain burden, ${ }^{c}$ severity-weighted hours } \\
\hline $\begin{array}{l}\text { Mean } \pm \text { SD } \\
\text { Median (interquartile } \\
\text { range) }\end{array}$ & $\begin{array}{l}41.5 \pm 39.3 \\
36.0(19.5-48.0)\end{array}$ & $\begin{array}{l}43.3 \pm 44.0 \\
28.0(13.5-54.0)\end{array}$ \\
\hline
\end{tabular}

Notes: Results are expressed as mean \pm SD or $n(\%)$ unless otherwise indicated. A significant difference was not observed between the treatment groups $(p>0.05)$. ${ }^{a}$ Number of times per week. bMeasured on a 5 -point scale, where $0=$ no pain, $\mathrm{I}=$ mild pain, $2=$ =moderate pain, $3=$ severe pain, $4=$ =very severe pain. ${ }^{\mathrm{C}}$ Calculated as the daily frequency of attacks $x$ the average pain severity of attacks in a day $x$ the average duration of attacks (in hours) in a day. This value was summed over 7 days to give the weekly total pain burden.

Abbreviations: $\mathrm{CH}$, cluster headache; s.c., subcutaneous; $\mathrm{SD}$, standard deviation.

\section{Individual Pain Components}

The overall LS mean reduction from baseline in average weekly $\mathrm{CH}$ attack frequency across weeks $1-3$ was significantly greater with galcanezumab than with placebo (Figure 2A). The overall LS mean reduction from baseline in weekly average duration of $\mathrm{CH}$ attacks across weeks $1-$ 3 was numerically greater in the galcanezumab group than in the placebo group, but not statistically significant (Figure 2B). The mean duration per attack was 0.12 hours (7.2 minutes) less for patients taking galcanezumab than for those taking placebo (95\% CI: 0.07, 0.32; $\mathrm{p}=0.20$ ). Similarly, the overall LS mean reduction from baseline in total weekly average $\mathrm{CH}$ attack severity over weeks 1-3 was numerically but not significantly greater with galcanezumab than with placebo (Figure 2C).

\section{Composite Score}

A significantly greater reduction in average weekly total pain burden was observed with galcanezumab compared 

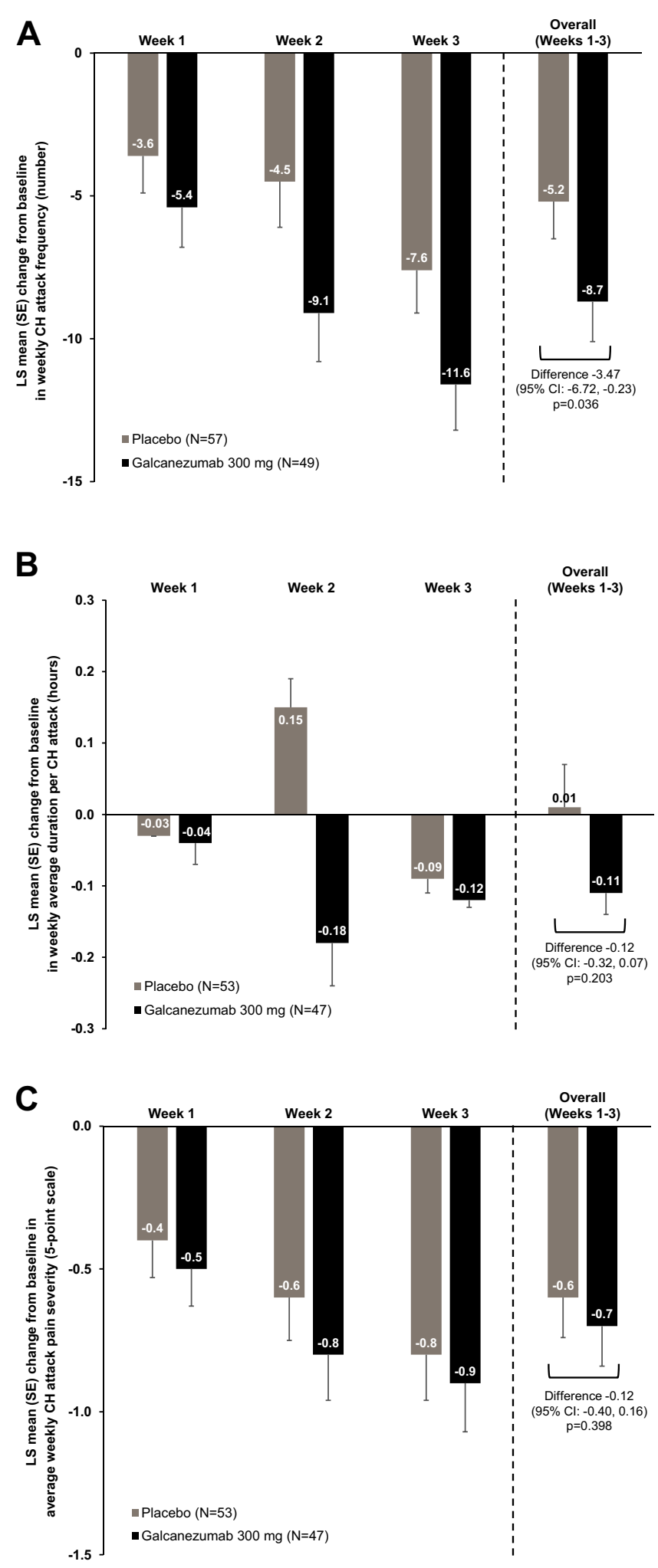

Figure $\mathbf{2}$ LS mean change from baseline in average weekly CH attack (A) frequency (primary study endpoint), (B) duration, and (C) severity, across weeks I-3 in patients receiving galcanezumab or placebo. Results are expressed with $\mathrm{SE}$; $\mathrm{p}$-values are from repeated measures analysis. $\mathbf{N}$ for $(\mathbf{A})$ are greater than those for $(\mathbf{B}$ and $\mathbf{C})$ because the analyses presented in (B and $\mathbf{C}$ ) were performed in patients with at least I week with at least one $\mathrm{CH}$ attack; consequently, a few patients who had zero attacks were excluded.

Abbreviations: $\mathrm{CH}$, cluster headache; $\mathrm{Cl}$, confidence interval; LS, least squares; $\mathrm{SE}$, standard error. 

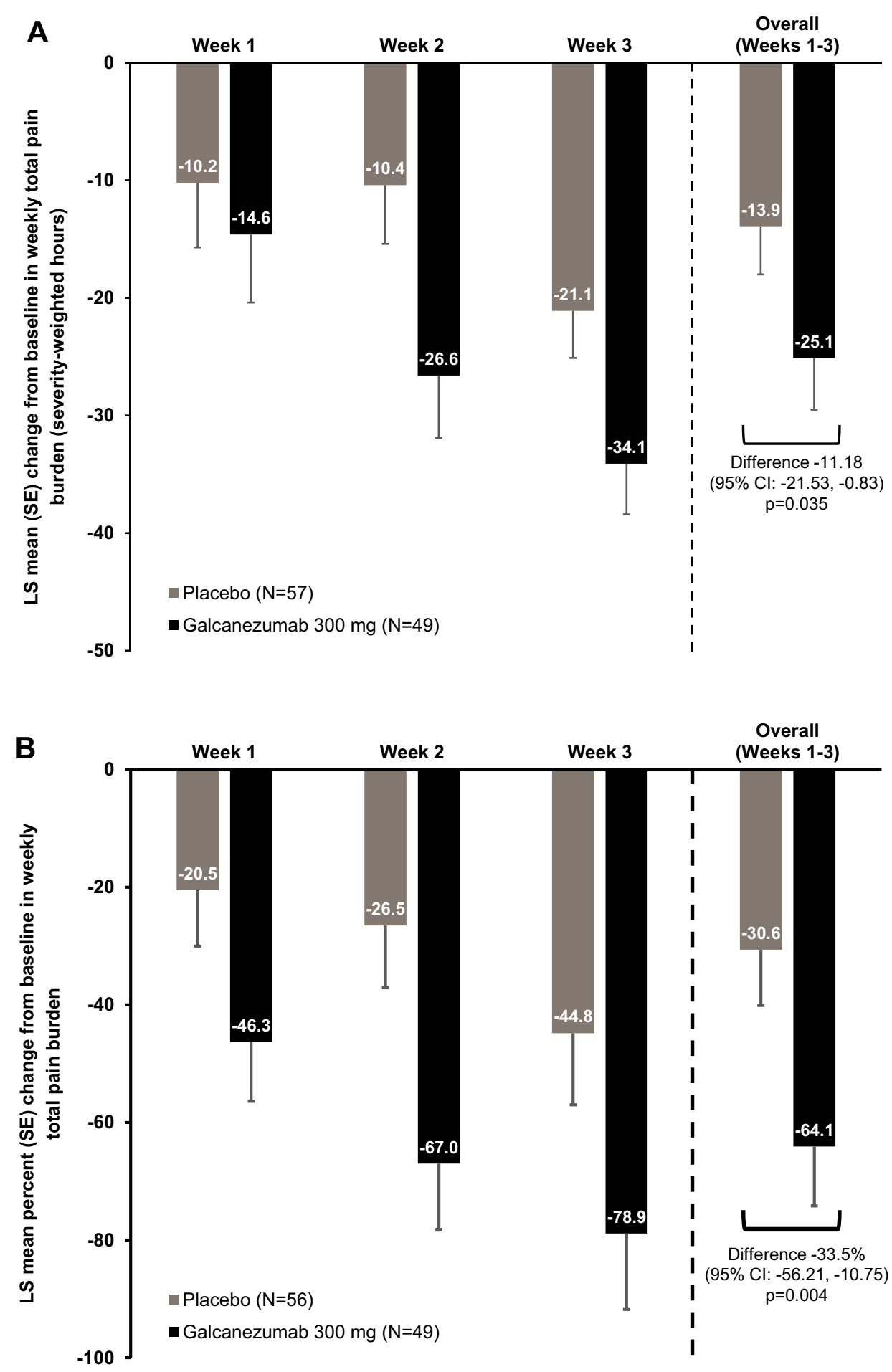

Figure 3 (A) LS mean change and (B) LS mean percent change from baseline in average weekly $\mathrm{CH}$ attack total pain burden across weeks I-3 in patients receiving galcanezumab or placebo. Results are expressed with SE; $\mathrm{P}$-values are from repeated measures analysis.

Abbreviations: $\mathrm{CH}$, cluster headache; $\mathrm{Cl}$, confidence interval; $\mathrm{LS}$, least squares; SE, standard error.

with placebo across weeks 1-3 (Figure 3A). The LS mean change (decrease) from baseline in weekly total pain burden across weeks $1-3$ was 11.18 severity-weighted hours greater for galcanezumab than for placebo $(95 \%$ CI for mean difference: $0.83,21.53 ; \mathrm{p}=0.035)$. Similarly, the LS mean percent change (decrease) from baseline in weekly total pain burden across weeks 1-3 was significantly greater with galcanezumab than placebo $(64 \%$ vs $31 \%$, respectively; mean difference $33.5 \%$, 95\% CI: 10.75, 56.21; $\mathrm{p}=0.004$ ) (Figure 3B). 


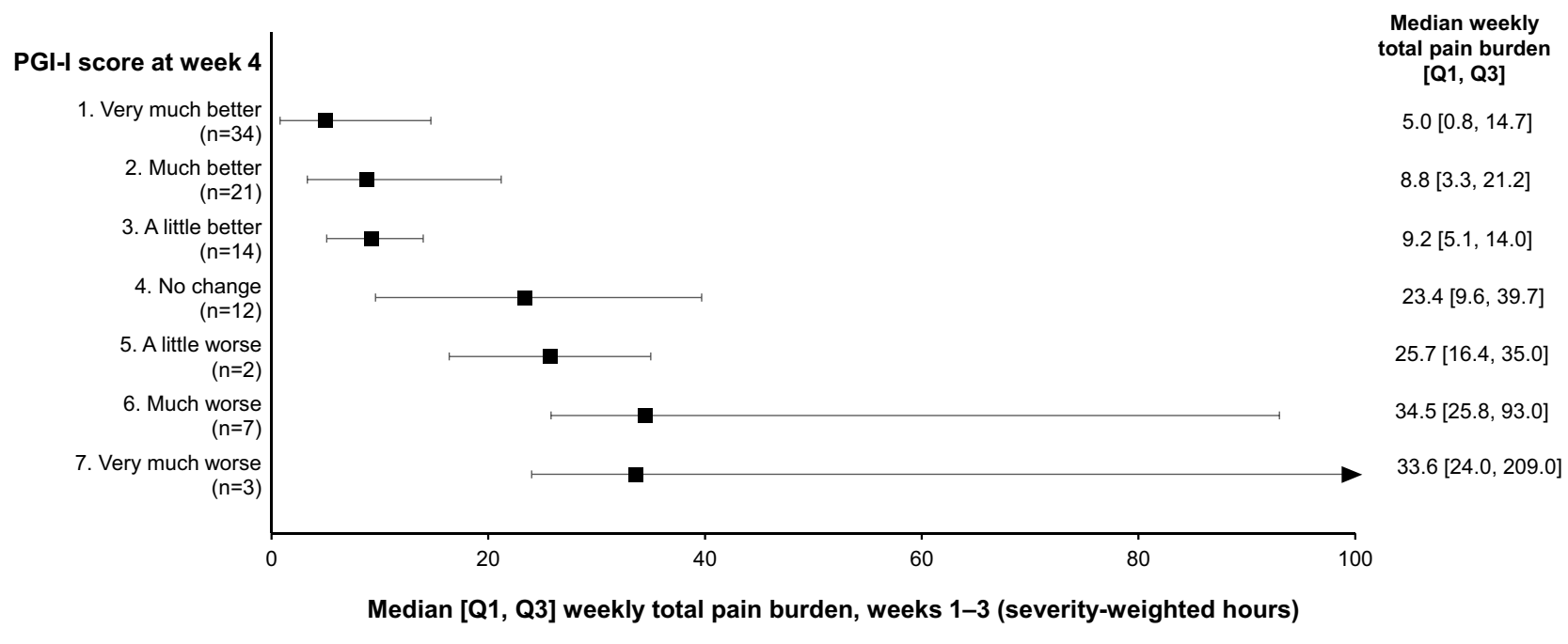

Figure 4 Median [QI, Q3] weekly total pain burden across weeks I-3 according to PGI-I score at week 4 in patients with episodic CH receiving galcanezumab or placebo $(\mathrm{N}=93)$.

Abbreviations: $\mathrm{CH}$, cluster headache; PGI-I, Patient Global Impression of Improvement; QI, quartile I; Q3, quartile 3.

The mean reduction from baseline in total pain burden was also significantly greater with galcanezumab than with placebo in each of the two sensitivity analyses that adjusted for weighted attack severity scores. Using the square root of attack severity to weight $\mathrm{CH}$ attack duration, the LS mean reduction from baseline in weekly total pain burden across weeks 1-3 was 14.74 (SE 2.51) severity-weighted hours with galcanezumab versus 8.77 (SE 2.35) severity-weighted hours with placebo (mean difference 5.96 [SE 2.98] severity-weighted hours; 95\% CI: $0.03,11.90 ; p=0.049$ ). Further, using the square of attack severity to weight $\mathrm{CH}$ attack duration, the corresponding mean change from baseline was 75.44 (SE 15.10) severityweighted hours with galcanezumab versus 35.34 (SE 14.13) severity-weighted hours with placebo (mean difference 40.09 [SE 17.57] severity-weighted hours; 95\% CI: $5.08,75.10 ; \mathrm{p}=0.025)$.

\section{Construct Validity}

The median weekly total pain burden across weeks 1-3 measured in all patients (pooled data) decreased as week 4 PGI-I ratings improved, from 33.6 severity-weighted hours for patients who reported being "very much worse" $(\mathrm{n}=3)$ to 5.0 severity-weighted hours for patients who reported feeling "very much better" ( $n=34)$ (Figure 4). The median (quartile 1, quartile 3) weekly total pain burden across weeks 1-3 was 7.9 (2.8, 14.7) severity-weighted hours for patients who reported feeling better ("a little better," "much better," or "very much better") at week 4 compared with 33.5 (19.8, 42.3) severity-weighted hours for patients who did not feel better ("no change," "a little worse," "much worse," or "very much worse"). Further, the odds of a patient reporting a PGII score of 1, 2, or 3 ("a little better," "much better," or "very much better") increased by an estimated $21 \%$ for every 10 severity-weighted hours reduction in total pain burden across weeks 1-3 (OR 1.21; 95\% CI: 1.05, 1.39; $\mathrm{p}=0.01$ ).

\section{Contribution of Individual Pain Components to Total Pain Burden}

Weekly total pain burden showed a moderate to high correlation with each of the three individual pain components at baseline: Spearman's rank correlation was 0.62 for weekly $\mathrm{CH}$ attack frequency, 0.64 for weekly average attack duration, and 0.49 for weekly average attack severity. The squared semi-partial type II correlation between total pain burden and average weekly $\mathrm{CH}$ attack frequency, duration, and severity was $0.33,0.43$, and 0.08 , respectively.

Mean change from baseline in mean weekly total pain burden across weeks 1-3 was numerically greater with galcanezumab than with placebo after adjusting for change from baseline in weekly $\mathrm{CH}$ attack frequency: the mean (SE) reduction in total pain burden was 21.42 (3.30) severity-weighted hours for galcanezumab compared with 16.62 (3.09) severity-weighted hours for placebo (mean difference 4.80; 95\% CI: 2.89, 12.48; $\mathrm{p}=0.22$ ).

\section{Discussion}

Results of the post hoc analyses reported here showed that treatment with galcanezumab significantly reduced the 
average total pain burden across weeks 1-3 compared with placebo in patients with episodic $\mathrm{CH}$ when assessed using a composite measure of three pain dimensions (attack frequency, duration, and severity). The results also suggested that the total pain burden score has construct validity, with the average weekly total pain burden across weeks 1-3 decreasing as week 4 PGI-I ratings improved.

As reflected in the squared semi-partial correlations, all three pain dimensions contributed to variability in the total pain burden score. However, attack frequency and duration provided a greater contribution to the total pain burden score than attack severity. Further, after adjusting for the effect of change from baseline in weekly $\mathrm{CH}$ attack frequency, galcanezumab showed some benefit in numerically reducing the remaining total pain burden. These analyses suggest that the reduction in total pain burden score may have been due to a reduction in either average attack duration or average attack severity in addition to the reduction in attack frequency. In other words, the total pain burden may represent an estimate of the overall burden of pain experienced by the patient that is not captured in the frequency of attacks alone. However, given the lack of statistical significance and the post hoc nature of these analyses, these results should be interpreted with caution.

Pain is a subjective, personal experience and has multiple dimensions, such as quality, location, intensity (severity), frequency, duration, and emotional and psychological impact. ${ }^{34-36}$ As a result, it can be difficult to measure. Acute pain and pain due to trauma is relatively straightforward to evaluate, but the assessment of persistent or complex pain is more challenging. ${ }^{37}$ Many different measurement tools have been used to evaluate pain in clinical research studies. ${ }^{34-36}$ Most commonly, the severity of pain is measured using self-report on a visual analog scale (VAS) or numeric rating scale (NRS) from 0 (no pain) to 10 (worst possible pain). In $\mathrm{CH}$ specifically, clinical trials of triptans (sumatriptan and zolmitriptan) for the acute treatment of $\mathrm{CH}$ have tended to measure the severity of pain using a 5-point scale of none, mild, moderate, severe, and very severe, ${ }^{38-43}$ whereas studies of preventive treatments for $\mathrm{CH}$ (eg, steroids and verapamil) have tended to measure the frequency of attacks. ${ }^{44,45}$

However, these approaches to pain measurement only provide a one-dimensional indication of pain; they do not reflect other dimensions of pain, such as duration, character (eg, hot/cold, crushing, tearing, cramping, stabbing, burning), and quality. ${ }^{34,46,47}$ It is recommended that patients are asked to describe pain according to a number of characteristics, including the severity, frequency, and duration. ${ }^{34,36,47}$ Examples of tools for measuring the multidimensional aspects of pain include the McGill Pain Questionnaire $^{48}$ and the Brief Pain Inventory. ${ }^{49}$ The evaluation of multiple pain dimensions can help to improve pain management and pain care outcomes and is also essential for assessing the effectiveness of treatments in clinical trials. ${ }^{37,50}$

In this study, the average total pain burden of $\mathrm{CH}$ over 3 weeks was measured using a composite of three pain dimensions (frequency, duration, and severity). Other studies have used composite pain measures, in both pain and primary headache disorders, including $\mathrm{CH}$. In a study of pediatric patients with chronic pain, for example, the authors developed a composite scale (the pain-frequency-severityduration scale) that, as the name indicates, measures the frequency, severity, and duration of pain to give a single composite score. The study revealed a significant correlation between this composite score, functional ability, and healthrelated quality of life. ${ }^{51}$ Similarly, researchers investigating opioid treatment in patients with intractable headache successfully used the Severe Headache Index, which incorporated both the frequency and the severity of headaches. ${ }^{52}$

In $\mathrm{CH}$, a study of patients receiving hyperbaric oxygen treatment for preventive treatment of the disease assessed the frequency and severity of headaches by calculating a headache index (sum of [the number of attacks $\times$ the degree of severity]). ${ }^{53}$ More recently, the Cluster Headache Severity Scale was developed, which includes the three dimensions of attack frequency per day, attack duration (in minutes), and duration of the $\mathrm{CH}$ period (in months). This provides a single score ranging from 3 to 12 but does not include a measure of attack severity. ${ }^{54}$ A matched-control cohort study used the Cluster Headache Index, which incorporates daily $\mathrm{CH}$ attack frequency and duration as well as the duration of each cluster period and the yearly cluster frequency. ${ }^{55}$

Galcanezumab is the first anti-CGRP agent that reduces the frequency of headache attacks to be approved by the FDA for the treatment of episodic $\mathrm{CH}^{22}$ Results of the current post hoc analyses suggest that galcanezumab also reduces the total pain burden of this disease when using a composite measure of three pain dimensions (attack frequency, duration, and severity).

\section{Study Strengths and Limitations}

The analyses reported here were based on a well-designed primary randomized controlled study with washout and follow-up periods. The study involved measurement of 
PROs, specifically measurement of three dimensions of pain using a daily headache e-diary. The use of an e-diary for the measurement of headache has been validated. $^{31}$ The main limitations of our analyses were their post hoc nature and the relatively small patient numbers; consequently, patients included in the analyses may not fully represent the broader population of patients with episodic $\mathrm{CH}$ who receive care in the "real world". In addition, the scoring of pain severity on a scale of $0-4$ was arbitrary, no adjustments for multiplicity were made, and PGI-I responses were measured at week 4, whereas parameters for calculating the total pain burden were measured across weeks 1-3. Thus, caution should be used when interpreting the results. We should also acknowledge that patients were allowed to take acute treatments, such as high-flow oxygen and subcutaneous sumatriptan, throughout the study. Use of these medications may impact aspects of episodic $\mathrm{CH}$ total pain burden, such as attack duration and, potentially, attack severity. However, use of acute medications, including high-flow oxygen and subcutaneous sumatriptan, was similar between the galcanezumab and placebo groups at baseline, suggesting that the reduction in episodic $\mathrm{CH}$ total pain burden with galcanezumab is particularly meaningful.

\section{Conclusions}

The reduction in average total pain burden associated with episodic $\mathrm{CH}$ across weeks 1-3 was significantly greater with galcanezumab than with placebo when measured using a composite of three pain dimensions (attack frequency, duration, and severity). Attack frequency and duration provided a greater contribution than attack severity to the total pain burden score. Patients who reported feeling better on the PGI-I at week 4 showed a significantly lower average total pain burden than patients who did not feel better, suggesting construct validity of the composite measure, but this requires further validation. Measurement of total pain burden may provide a holistic approach to describing the pain experienced by patients with episodic $\mathrm{CH}$.

\section{Data Sharing Statement}

Lilly provides access to all individual participant data collected during the trial, after anonymization, with the exception of pharmacokinetic or genetic data. Data are available to request 6 months after the indication studied has been approved in the US and EU and after primary publication acceptance, whichever is later. No expiration date of data requests is currently set once data are made available.
Access is provided after a proposal has been approved by an independent review committee identified for this purpose and after receipt of a signed data sharing agreement. Data and documents, including the study protocol, statistical analysis plan, clinical study report, blank or annotated case report forms, will be provided in a secure data sharing environment. For details on submitting a request, see the instructions provided at www.vivli.org.

\section{Acknowledgments}

This post hoc analysis and study NCT02397473 were sponsored by Eli Lilly and Company.

Medical writing assistance was provided by Dr Sue Chambers and Caroline Spencer ( $\mathrm{Rx}$ Communications, Mold, UK) and was funded by Eli Lilly and Company.

\section{Disclosure}

M Rettiganti, T Oakes, JN Bardos, R Wenzel, DK Kuruppu, and JM Martinez are employees and stockholders of Eli Lilly and Company and/or one of its subsidiaries. JS Andrews was an employee at Eli Lilly at the time the study was conducted and is currently employed at Takeda Pharmaceuticals. D Kudrow has received personal compensation for speaking or for serving on an advisory board from Amgen, Alder Biopharmaceuticals, Biohaven Pharmaceuticals, Eli Lilly and Company, Axsome, Allergan, Lundbeck, Satsuma, Teva Pharmaceutical Industries Ltd, and Xoc Pharmaceuticals and has received research support from Amgen, Alder Biopharmaceuticals, Biogen, Biohaven Pharmaceuticals, Eli Lilly and Company, Roche-Genentech, Teva Pharmaceutical Industries Ltd, and VM Biopharma. C Gaul has received honoraria for consulting and lectures within the past 3 years from Allergan, Grünenthal, Hormosan Pharma, Eli Lilly and Company, Lundbeck, Novartis, Reckitt Benckiser, Sanofi Aventis, and Teva Pharmaceutical Industries Ltd and does not hold any stocks of pharmaceutical or medical device companies. He is honorary secretary of the German Migraine and Headache Society. The authors report no other conflicts of interest in this work.

\section{References}

1. Headache Classification Committee of the International Headache Society (IHS). The international classification of headache disorders, 3rd edition. Cephalalgia. 2018;38(1):1-211. doi:10.1177/03331024 17738202

2. Matharu MS, Goadsby PJ. Trigeminal autonomic cephalalgias: diagnosis and management. In: Silberstein SD, Lipton RB, Dodick DW, editors. Wolff's Headache and Other Head Pain. 8th ed. New York, NY: Oxford University Press; 2008:379-430. 
3. Abu Bakar N, Tanprawate S, Lambru G, et al. Quality of life in primary headache disorders: a review. Cephalalgia. 2016;36:67-91. doi:10.1177/0333102415580099

4. Hoffmann J, May A. Diagnosis, pathophysiology, and management of cluster headache. Lancet Neurol. 2018;17(1):75-83. doi:10.1016/ S1474-4422(17)30405-2

5. Rozen TD, Fishman RS. Cluster headache in the United States of America: demographics, clinical characteristics, triggers, suicidality, and personal burden. Headache. 2012;52(1):99-113. doi:10.1111/ j.1526-4610.2011.02028.x

6. Lee MJ, Cho SJ, Park JW, et al. Increased suicidality in patients with cluster headache. Cephalalgia. 2019;39(10):1249-1256. doi:10.1177/ 0333102419845660

7. Gaul C, Finken J, Biermann J, et al. Treatment costs and indirect costs of cluster headache: a health economics analysis. Cephalalgia. 2011;31(16):1664-1672. doi:10.1177/0333102411425866

8. Ford JH, Nero D, Kim G, et al. Societal burden of cluster headache in the United States: a descriptive economic analysis. J Med Econ. 2018;21(1):107-111. doi:10.1080/13696998.2017.1404470

9. Polson M, Lord TC, Evangelatos TM, et al. Real-world health plan claims analysis of differences in healthcare utilization and total costs in patients suffering from cluster headaches and those without headache-related conditions. Am J Manag Care. 2017;23(16 suppl): S295-S299.

10. Choi YJ, Kim BK, Chung PW, et al. Impact of cluster headache on employment status and job burden: a prospective cross-sectional multicenter study. J Headache Pain. 2018;19(1):78. doi:10.1186/ s10194-018-0911-x

11. British Association for the Study of Headache. Guidelines for all healthcare professionals in the diagnosis and management of migraine, tension-type, cluster and medication-overuse headache. 3rd edition; 2010. Available from: http://www.bash.org.uk/wpcontent/uploads/2012/07/10102-BASH-Guidelines-update-2_v51-indd.pdf. Accessed August 1, 2019.

12. Sarchielli P, Granella F, Prudenzano MP, et al. Italian guidelines for primary headaches: 2012 revised version. J Headache Pain. 2012;13 (Suppl 2):S31-S70.

13. May A, Leone M, Afra J, et al. EFNS guidelines on the treatment of cluster headache and other trigeminal-autonomic cephalalgias. Eur J Neurol. 2006;13(10):1066-1077. doi:10.1111/j.1468-1331.2006.01 566.X

14. Becker WJ, Findlay T, Moga C, et al. Guideline for primary care management of headache in adults. Can Fam Physician. 2015;61 (8):670-679.

15. National Institute for Health and Care Excellence. Headaches in over 12s: diagnosis and management. Clinical guideline [CG150]; 2015. Available from: https://www.nice.org.uk/guidance/cg150. Accessed August 5, 2019.

16. Robbins MS, Starling AJ, Pringsheim TM, et al. Treatment of cluster headache: the American Headache Society evidence-based guidelines. Headache. 2016;56(7):1093-1106. doi:10.1111/head.12 866

17. Goadsby PJ, Edvinsson L, Ekman R. Vasoactive peptide release in the extracerebral circulation of humans during migraine headache. Ann Neurol. 1990;28(2):183-187. doi:10.1002/ana.410280213

18. Goadsby PJ, Edvinsson L. Human in vivo evidence for trigeminovascular activation in cluster headache. Neuropeptide changes and effects of acute attacks therapies. Brain. 1994;117(3):427-434. doi:10.1093/brain/117.3.427

19. Benschop RJ, Collins EC, Darling RJ, et al. Development of a novel antibody to calcitonin gene-related peptide for the treatment of osteoarthritis-related pain. Osteoarthr Cartilage. 2014;22(4):5 78-585. doi:10.1016/j.joca.2014.01.009

20. Edvinsson L. The trigeminovascular pathway: role of CGRP and CGRP receptors in migraine. Headache. 2017;57:47-55. doi:10. 1111/head.13081
21. Vollesen ALH, Snoer A, Beske RP, et al. Effect of infusion of calcitonin gene-related peptide on cluster headache attacks. JAMA Neurol. 2018;75(10):1187-1197. doi:10.1001/jamaneurol.2018.1675

22. Food and Drug Administration. Drug approval package: Emgality (galcanezumab-gnlm); 2019. Available from: https://www.access data.fda.gov/drugsatfda docs/label/2019/761063s003lbl.pdf. Accessed January 22, 2021.

23. Detke HC, Goadsby PJ, Wang S, Friedman DI, Selzler KJ, Aurora SK. Galcanezumab in chronic migraine: the randomized, double-blind, placebo-controlled REGAIN study. Neurology. 2018;91(24):e2211-e2221. doi:10.1212/WNL.0000000000006640

24. Food and Drug Administration. Drug approval package: Emgality (galcanezumab-gnlm); 2018. Available from: https://www.access data.fda.gov/drugsatfda_docs/label/2018/761063s000lbl.pdf. Accessed January 22, 2021.

25. Förderreuther S, Zhang Q, Stauffer VL, et al. Preventive effects of galcanezumab in adult patients with episodic or chronic migraine are persistent: data from the phase 3, randomized, double-blind, placebo-controlled EVOLVE-1, EVOLVE-2, and REGAIN studies. J Headache Pain. 2018;19(1):121. doi:10.11 86/s10194-018-0951-2

26. Stauffer VL, Dodick DW, Zhang Q, et al. Evaluation of galcanezumab for the prevention of episodic migraine: the EVOLVE-1 randomized clinical trial. JAMA Neurol. 2018;75(9):1080-1088. doi:10. 1001/jamaneurol.2018.1212

27. Skljarevski V, Matharu M, Millen BA, et al. Efficacy and safety of galcanezumab for the prevention of episodic migraine: results of the EVOLVE-2 phase 3 randomized controlled clinical trial. Cephalalgia. 2018;38(8):1442-1454. doi:10.1177/0333102418779 543

28. Silberstein SD, Stauffer VL, Day KA, et al. Galcanezumab in episodic migraine: subgroup analyses of efficacy by high versus low frequency of migraine headaches in phase 3 studies (EVOLVE-1 \& EVOLVE-2). J Headache Pain. 2019;20(1):75. doi:10.1186/s10194019-1024-X

29. Goadsby PJ, Dodick DW, Leone M, et al. Trial of galcanezumab in prevention of episodic cluster headache. $N$ Engl J Med. 2019;381 (2):132-141. doi:10.1056/NEJMoa1813440

30. Niere K, Jerak A. Measurement of headache frequency, intensity and duration: comparison of patient report by questionnaire and headache diary. Physiother Res Int. 2004;9(4):149-156. doi:10.1002/pri.318

31. Bandarian-Balooch S, Martin PR, McNally B, et al. Electronic diary for recording headaches, triggers, and medication use: development and evaluation. Headache. 2017;57(10):1551-1569. doi:10.1111/ head. 13184

32. Lemmens J, De Pauw J, Van Soom T, et al. The effect of aerobic exercise on the number of migraine days, duration and pain intensity in migraine: a systematic literature review and meta-analysis. J Headache Pain. 2019;20(1):16. doi:10.1186/s10194-019-0961-8

33. Headache Classification Committee of the International Headache Society (IHS). The international classification of headache disorders, 3rd edition (beta version). Cephalalgia. 2013;33(9):629-808. doi:10.1177/0333102413485658

34. Caraceni A, Cherny N, Fainsinger R, et al. Pain measurement tools and methods in clinical research in palliative care: recommendations of an Expert Working Group of the European Association of Palliative Care. J Pain Symptom Manage. 2002;23:239-255. doi:10.1016/S0885-3924(01)00409-2

35. Dworkin RH, Turk DC, Farrar JT, et al. Core outcome measures for chronic pain clinical trials: IMMPACT recommendations. Pain. 2005;113(1-2):9-19. doi:10.1016/j.pain.2004.09.012

36. Hjermstad MJ, Gibbins J, Haugen DF, et al. Pain assessment tools in palliative care: an urgent need for consensus. Palliat Med. 2008;22:895-903. doi:10.1177/0269216308095701

37. Breivik H, Borchgrevink PC, Allen SM, et al. Assessment of pain. $\mathrm{Br}$ $J$ Anaesth. 2008;101(1):17-24. doi:10.1093/bja/aen103 
38. Pilgrim AJ. Methodology of clinical trials of sumatriptan in migraine and cluster headache. Eur Neurol. 1991;31(5):295-299. doi:10.1159/ 000116757

39. Sumatriptan Cluster Headache Study Group. Treatment of acute cluster headache with sumatriptan. $N$ Engl J Med. 1991;325 (5):322-326. doi:10.1056/NEJM199108013250505

40. Van Vliet JA, Bahra A, Martin V, et al. Intranasal sumatriptan in cluster headache: a randomized placebo-controlled double-blind study. Neurology. 2003;60(4):630-633. doi:10.1212/01.WNL.0000046589.45 855.30

41. Cittadini E, May A, Straube A, et al. Effectiveness of intranasal zolmitriptan in acute cluster headache: a randomized, placebo-controlled, double-blind crossover study. Arch Neurol. 2006;63:1537-1542. doi:10.1001/archneur.63.11.nct60002

42. Rapoport AM, Mathew MT, Silberstein SD, et al. Zolmitriptan nasal spray in the acute treatment of cluster headache: a double-blind study. Neurology. 2007;69:821-826. doi:10.1212/01.wnl.0000267886.85 210.37

43. Hedlund C, Rapoport AM, Dodick DW, Goadsby GJ. Zolmitriptan nasal spray in the acute treatment of cluster headache: a meta-analysis of two studies. Headache. 2009;49(9):1315-1323. doi:10.1111/j.1526-4610.2009.01518.x

44. Leone M, D'Amico D, Frediani F, et al. Verapamil in the prophylaxis of episodic cluster headache: a double-blind study versus placebo. Neurology. 2000;54(6):1382-1385. doi:10.1212/WNL.54.6.1382

45. Holle D, Burmeister J, Scherag A, et al. Study protocol of Prednisone in episodic Cluster Headache $(\mathrm{PredCH})$ : a randomized, double-blind, placebo-controlled parallel group trial to evaluate the efficacy and safety of oral prednisone as an add-on therapy in the prophylactic treatment of episodic cluster headache with verapamil. BMC Neurol. 2013;13:99. doi:10.1186/1471-2377-13-99

46. Van Boekel RLM, Vissers KCP, van der Sande R, et al. Moving beyond pain scores: multidimensional pain assessment is essential for adequate pain management after surgery. PLoS One. 2017;12(5): e0177345. doi:10.1371/journal.pone.0177345
47. Kishner S. Pain assessment. Medscape article 1948069; 2018. Available from: https://emedicine.medscape.com/article/1948069overview. Accessed August 5, 2019.

48. Main CJ. Pain assessment in context: a state of the science review of the McGill pain questionnaire 40 years on. Pain. 2016;157 (7):1387-1399. doi:10.1097/j.pain.0000000000000457

49. Poquet N, Lin C. The Brief Pain Inventory (BPI). J Physiother. 2016;62(1):52. doi:10.1016/j.jphys.2015.07.001

50. Petti E, Scher C, Meador L, et al. Can multidimensional pain assessment tools help improve pain outcomes in the perianesthesia setting? $J$ Perianesth Nurs. 2018;33(5):767-772. doi:10.1016/j.jopan.2018. 07.010

51. Salamon KS, Davies WH, Fuentes MR, et al. The pain frequency-severity-duration scale as a measure of pain: preliminary validation in a pediatric chronic pain sample. Pain Res Treat. 2014;2014:653592. doi:10.1155/2014/653592

52. Saper JR, Lake AE, Hamel RL, et al. Daily scheduled opioids for intractable head pain: long-term observations of a treatment program. Neurology. 2004;62(10):1687-1694. doi:10.1212/01.WNL.00001251 89.17830 .02

53. Nilsson Remahl AI, Ansjon R, Lind F, Waldenlind E. Hyperbaric oxygen treatment of active cluster headache: a double-blind placebo-controlled cross-over study. Cephalalgia. 2002;22:730-739. doi:10.1046/j.1468-2982.2002.00450.x

54. Steinberg A, Fourier C, Ran C, et al. Cluster headache - clinical pattern and a new severity scale in a Swedish cohort. Cephalalgia. 2018;38:1286-1295. doi:10.1177/0333102417731773

55. Barloese M, Lund N, Petersen A, Rasmussen M, Jennum P, Jensen R. Sleep and chronobiology in cluster headache. Cephalagia. 2015;35 (11):969-978. doi:10.1177/0333102414564892
Journal of Pain Research

\section{Publish your work in this journal}

The Journal of Pain Research is an international, peer reviewed, open access, online journal that welcomes laboratory and clinical findings in the fields of pain research and the prevention and management of pain. Original research, reviews, symposium reports, hypothesis formation and commentaries are all considered for publication. The manuscript management system is completely online and includes a very quick and fair peer-review system, which is all easy to use. Visit http:// www.dovepress.com/testimonials.php to read real quotes from published authors. 